\title{
The Probabilistic Serial Mechanism with Private Endowments
}

\author{
Özgür Yılmaz *† \\ College of Administrative Science and Economics, Koç University, Sarıyer, İstanbul, Turkey 34450
}

April 2009

\begin{abstract}
A random assignment is ordinally efficient if it is not stochastically dominated with respect to individual preferences over sure objects. When there are no private endowments, the set of ordinally efficient random assignments is characterized by the eating algorithm (Bogomolnaia and Moulin 2001). However, when there are private endowments, the main requirement is individual rationality and and the eating algorithm fails to deliver this property. Our contribution is the natural generalization of the eating algorithm for this general class of problems. The family of this generalized eating algorithm characterizes the set of individually rational and ordinally efficient random assignments. A special solution in this family, the individually rational probabilistic serial ( $\left.\mathrm{PS}^{\mathbf{I R}}\right)$, also achieves a new fairness axiom, no justified-envy. However, it is not immune to strategic manipulation. We show that individual rationality, no justified-envy and strategy-proofness are incompatible.
\end{abstract}

Keywords : random assignment, no justified-envy, ordinal efficiency, simultaneous eating algorithm

Journal of Economic Literature Classification Numbers: C71, C78, D71, DF8

*E-mail address: ozyilmaz@ku.edu.tr; Phone: +90 212338 1627; Fax: +90 2123381653.

${ }^{\dagger}$ I am particularly indebted to William Thomson for his guidance and support. I would like to thank to Diego Dominguez, Hervé Moulin, Jay Sethuraman, Utku Ünver and especially Tayfun Sönmez for their useful comments. 


\section{Introduction}

An assignment problem is a resource allocation problem where a set of objects has to be allocated to a group of agents in such a way that each agent receives at most one object; monetary transfers between the agents are not permitted. This class of problems occupies a special place in the literature due to its strong empirical relevance. Examples include the assignment of campus housing to students, jobs to workers, rooms to housemates, and offices to professors. For convenience, this paper uses language that fits the first example and refers to the objects as houses.

Three special subclasses of assignment problems can be distinguished depending on who owns the houses. In an assignment problem with private endowments, each agent owns a particular house. In an assignment problem with a social endowment, agents own houses collectively. ${ }^{1}$ The focus of the current paper is on the third class, which is a mixture of the first two: Some agents (existing tenants) own their own houses whereas others (new applicants) do not, and the houses not owned by the existing tenants are the social endowment. Following Abdulkadiroğlu and Sönmez (1999), we refer to this problem as a house allocation problem with existing tenants. Our goal is to propose an efficient and fair solution to this class of assignment problems.

In assignment problems with private endowments, one of the main requirements that one may want to impose is individual rationality: each agent finds his assignment at least as desirable as his endowment. An individually rational solution is the top trading cycles (TTC) solution. The TTC solution is defined as follows: Each agent points to an agent (possibly himself). A top trading cycle consists of agents such that each agent in the cycle points to the next agent. Since the number of agents is finite, there is at least one cycle. Each agent in each cycle is assigned the house of the agent to whom he points. The agents in all cycles are removed with their assigned houses. The procedure is repeated until each agent receives a house.

In this class of problems, if preferences are strict, the core allocation is a singleton (Roth and Postlewaite 1977), and the TTC solution selects it. The core, thus the TTC solution, is strategy-proof (Roth 1982). In fact, it is the only solution that is individually rational, efficient, and strategy-proof (Ma 1994, Svensson 1999).

In assignment problems with a social endowment, treating equals equally is in-

\footnotetext{
${ }^{1}$ Assignment problems with a social endowment and private endowments are introduced by Hylland and Zeckhauser (1979) and Shapley and Scarf (1974), respectively. In the literature, these two classes of problems are referred to as house allocation problems and housing markets, respectively.
} 
dispensable and to achieve it, a lottery mechanism is commonly used in real life applications: An ordering of agents is randomly drawn from the uniform distribution. For a given ordering, the first agent chooses a house, then the second agent chooses a house among the remaining houses, and so on. This is the random priority (RP) solution. ${ }^{2}$ The RP solution is strategy-proof and it treats equals equally. Also, since, for each ordering, it selects an efficient allocation, the RP solution is ex-post efficient. However, when agents are equipped with von Neumann-Morgenstern preferences over random allocations (lotteries over assignments of houses), an impossibility result by Zhou (1990) implies that the RP solution is not ex-ante efficient. ${ }^{3}$ Actually, it does not satisfy ordinal efficiency, an efficiency requirement for ordinal mechanisms where only individual preferences over sure houses are elicited. ${ }^{4}$

Another class of solutions is proposed by Bogomolnaia and Moulin (2001): Each house is imagined as being infinitely divisible. There is one unit of each house. A quantity of house $h$, given to agent $i$, represents the probability with which agent $i$ is assigned house $h$. For each agent, an 'eating' speed is specified on the unit interval. At any time, each agent eats his favorite available house at the specified speed: if the houses $a, b, c, \ldots$ have been entirely eaten (one unit of each has been distributed), and houses $x, y, z, \ldots$ have not, each agent starts eating his favorite house among $x, y, z, \ldots$ This class of algorithms is referred to as the parametric family of eating algorithms ${ }^{5}$ and characterizes the set of ordinally efficient random assignments. This family defines a class of solutions. A special solution in this class is the probabilistic serial solution (PS), which requires each agent's eating speed to be the same and constant. The PS solution improves on the RP solution in terms of efficiency and fairness: it is ordinally efficient and envy-free. The weakness of the PS solution is that it only satisfies weak strategy-proofness.

The class of assignment problems with private endowments and a social endowment is a mixture of the first two, and the question is whether the solutions discussed above

\footnotetext{
${ }^{2}$ Abdulkadiroğlu and Sönmez (1998) show that the RP solution (they call it the random serial dictatorship) is equivalent to the 'core from random endowment' solution, a solution that randomly chooses an endowment profile and then selects the core of the induced assignment problem with private endowments.

${ }^{3}$ Zhou shows that there is no lottery mechanism that is ex-ante efficient, anonymous, and strategyproof.

${ }^{4}$ For ordinal efficiency and its analysis, see Bogomolnaia and Moulin (2001), Abdulkadiroğlu and Sönmez (2003), and McLennan (2002).

${ }^{5}$ See also Bogomolnaia and Moulin (2002), and Crès and Moulin (2001).
} 
extend to this general class of problems. (Note that for this general class of problems, the TTC solution is not well defined, and the RP and PS solutions are not individually rational.) Abdulkadiroğlu and Sönmez (1999) introduced a generalization of the TTC solution, TTC solution from random orderings, which reduces to the RP solution when there are no private endowments, and to the TTC solution when there is no social endowment. ${ }^{6}$ The TTC solution from random orderings is individually rational, ex-post efficient, and strategy-proof. ${ }^{7}$ On the other hand, how the eating algorithm extends so as to satisfy individual rationality has been an open question.

Our contribution is to fill this gap: we generalize the parametric family of eating algorithms to account for individual rationality. The family of this generalized eating algorithm characterizes the set of individually rational and ordinally efficient random assignments. The problem in generalizing the eating algorithm is that, while agents eat houses, individual rationality is possibly violated for an agent or for a group of agents. We develop a recursive algorithm to prevent these violations. Our algorithm defines a class of solutions; to show that each solution in this class satisfies individual rationality, we use an elegant result from graph theory, the Supply-Demand Theorem by Gale (1957). Moreover, a special solution in this class satisfies a new axiom of fairness, no justified-envy. This new axiom weakens no envy: ${ }^{8}$ it views an assignment as unfair if an agent does not prefer his consumption to another agent's consumption and the assignment obtained by swapping their consumptions respects the individual rationality requirement of the latter agent. We further show that individual rationality, strategy-proofness and no justified-envy are incompatible.

\section{The model}

A non-empty finite set of houses $H$ has to be allocated to a non-empty finite set of agents $I$ in such a way that each agent receives at most one house; monetary transfers

\footnotetext{
${ }^{6}$ Pápai (2000) introduced hierarchical exchange rules, which generalize TTC mechanism and includes TTC from random orderings as a special class.

${ }^{7}$ Recently, Sönmez and Ünver (2005) generalized the main result by Abdulkadiroğlu and Sönmez (1998) (see footnote 2): First, construct an endowment structure by assigning each existing tenant his own house and randomly assigning the vacant houses to new applicants with uniform distribution. The core based mechanism chooses the core allocation of the induced housing market. The core based mechanism is equivalent to an extreme case of the TTC mechanism where new applicants are randomly ordered first and existing tenants are randomly ordered next.

${ }^{8}$ We need an alternative notion of fairness because individual rationality is the key property when there are private endowments and it is incompatible with no envy, the central axiom of fairness.
} 
between agents are not permitted. Being unassigned to any of the houses in $H$ is denoted by $h_{0}$.

An endowment profile is a function $\mu^{0}: I \rightarrow H \cup\left\{h_{0}\right\}$ such that $\mu^{0}(i)=$ $\mu^{0}(j)$ implies $\mu^{0}(i)=h_{0}$. Let $\mathcal{M}^{0}$ denote the set of all endowment profiles. Given an endowment profile $\mu^{0} \in \mathcal{M}^{0}$, the sets $H_{O} \equiv\left\{\mu^{0}(i): i \in I\right\} \backslash\left\{h_{0}\right\}$ and $H_{V} \equiv H \backslash H_{O}$ are the sets of occupied and vacant houses respectively. Also, $I_{E} \equiv\left\{i \in I: \mu^{0}(i) \in H\right\}$ and $I_{N} \equiv\left\{i \in I: \mu^{0}(i)=h_{0}\right\}$ are the sets of existing tenants and new applicants, respectively. Each existing tenant $i$ is assumed to have the right of living in the house he occupies, $\mu^{0}(i) \in H_{O}$.

Each agent $i$ has a strict preference relation $R_{i}$ on $H$. We denote this domain of preferences by $\mathcal{D}$. Each agent prefers each house to $h_{0}$ and also, $|I|=|H|{ }^{9}$ Let $R=\left(R_{i}\right)_{i \in I}$ be a preference profile. Also, for each $S \subseteq I$, let $R_{S}=\left(R_{i}\right)_{i \in S}$.

A house allocation problem with existing tenants, or simply a problem, is a quadruple $\left(I, H, \mu^{0}, R\right)$. Since the sets $I$ and $H$ are fixed throughout the paper, we use $\left(\mu^{0}, R\right)$ instead of $\left(I, H, \mu^{0}, R\right)$ to denote a problem.

Given a problem $\left(\mu^{0}, R\right)$ and a house $h \in H$, let $U\left(R_{i}, h\right) \equiv\left\{h^{\prime} \in H: h^{\prime} R_{i} h\right\}$ be the upper contour set of $\mathbf{R}_{i}$ at $\mathbf{h}$. When there is no danger of confusion, we denote $U\left(R_{i}, \mu^{0}(i)\right)$ by $U_{i}$. Let

$$
U_{S} \equiv \bigcup_{i \in S} U_{i}
$$

A deterministic assignment is a bijection $\mu$ from $I$ into $H$. A deterministic assignment is represented as a permutation matrix, that is, a $|I| \times|H|$ matrix with entries 0 or 1 , and exactly one nonzero entry per row and one per column. Let $\mathcal{M}$ denote the set of all deterministic assignments. We extend the preference of agent $i$ to the set of deterministic assignments in the following natural way: Agent i prefers $\mu$ to $\mu^{\prime}$ if, and only if, he prefers $\mu(i)$ to $\mu^{\prime}(i)$.

A random consumption is a probability distribution over $H$. Let $\triangle H$ denote the set of all random consumptions. A lottery is a probability distribution over deterministic assignments. Let $\triangle \mathcal{M}$ denote the set of all lotteries. Each lottery induces a random assignment $Q=\left[q_{i h}\right]_{i \in I, h \in H}$, where $q_{i h} \in[0,1]$ is the probability that agent $i$ receives house $h$. Let $Q_{i}$ denote the resulting random consumption for agent $i$. A random assignment is represented as a bistochastic matrix. By the classical Birkhoff

\footnotetext{
${ }^{9}$ These assumptions are not critical; all our definitions and results extend verbatim to the case of opting out and the case of different number of agents and houses (Section 6).
} 
(1946) - von Neumann (1953) Theorem, every bistochastic matrix obtains as a (in general, not unique) convex combination of permutation matrices (deterministic assignments). Thus, every such matrix corresponds to at least one lottery. Since two lotteries resulting in the same bistochastic matrix yield the same random consumption to each agent, we will not distinguish them. Let $\mathcal{Q}$ denote the set of all random assignments. A solution is a function $\varphi: \mathcal{M}^{0} \times \mathcal{D}^{|I|} \rightarrow \mathcal{Q}$.

A deterministic assignment is individually rational if each existing tenant finds his assignment at least as desirable as his endowment. A solution is individually rational, if for each agent, the support of his random consumption is contained in the upper contour set of his preferences at his endowment.

A deterministic assignment is Pareto efficient if no other deterministic assignment makes each agent at least as well off and at least one agent better off. A lottery is ex post efficient if it gives positive weights only to Pareto efficient deterministic assignments.

Given $i \in I, R_{i} \in \mathcal{D}$, and a pair of random consumptions $Q_{i}$ and $T_{i}, \mathbf{Q}_{\mathbf{i}}$ stochastically dominates $\mathbf{T}_{\mathbf{i}}$ for agent $i$, written as $\mathbf{Q}_{\mathbf{i}} \mathbf{s d}\left(\mathbf{R}_{\mathbf{i}}\right) \mathbf{T}_{\mathbf{i}}$, if, and only if,

$$
\forall h \in H, \quad \sum_{h^{\prime} \in U\left(R_{i}, h\right)} q_{i h^{\prime}} \geq \sum_{h^{\prime} \in U\left(R_{i}, h\right)} t_{i h^{\prime}}
$$

Given a pair of distinct random assignments $Q$, and $T, \mathbf{Q}$ stochastically dominates $\mathbf{T}$, if, and only if, for each $i \in I, Q_{i}$ stochastically dominates $T_{i}$. A random assignment is ordinally efficient if, and only if, it is not stochastically dominated by any other random assignment. A solution is ordinally efficient if it always selects ordinally efficient random assignments.

Given a preference list $R=\left(R_{i}\right)_{i \in I}$, a random assignment $Q=\left[q_{i h}\right]_{i \in I, h \in H}$ is envyfree if, for each $i, j \in I, Q_{i}$ stochastically dominates $Q_{j}$ at $R_{i}$. A solution satisfies no envy if it always selects envy-free random assignments.

A solution is strategy-proof if truth-telling is a dominant strategy in its associated preference revelation game. A solution $\varphi: \mathcal{M}^{0} \times \mathcal{D}^{|I|} \rightarrow \mathcal{Q}$ is weakly strategy-proof if, for each $\left(\mu^{0}, R\right) \in\left(\mathcal{M}^{0} \times \mathcal{D}^{|I|}\right)$, each $i \in I$, and each $R_{i}^{*} \in \mathcal{D}$,

$$
\varphi_{i}\left(\mu^{0}, R_{i}^{*}, R_{-i}\right) s d\left(R_{i}\right) \varphi_{i}\left(\mu^{0}, R\right) \Rightarrow \varphi_{i}\left(\mu^{0}, R_{i}^{*}, R_{-i}\right)=\varphi_{i}\left(\mu^{0}, R\right)
$$




\section{The eating algorithm and individual rationality}

Bogomolnaia and Moulin (2001) proposed a new class of solutions to assignment problems with a social endowment. This class is defined by the parametric family of eating algorithms:

Each house is imagined as being infinitely divisible. There is one unit of each house. A quantity of house $h$, given to agent $i$, represents the probability with which agent $i$ is assigned house $h$. For each agent $i$, let $\omega_{i}:[0,1] \rightarrow \mathbb{R}_{+}$be a function such that $\int_{0}^{1} \omega_{i}(t) d t=1$. The function $\omega_{i}$ is called the eating speed function of agent $i$, and $\omega_{i}(t)$ is the eating speed of agent $i$ at time $t$. Given the profile of eating speeds $\omega=\left(\omega_{i}\right)_{i \in I}$ and the profile $R$ of preferences, at time $t$, the eating algorithm lets agent $i$ eat his favorite available house at the speed $\omega_{i}(t)$ : if at time $t$, the houses $a, b, c \ldots$ have been entirely eaten and houses $x, y, z \ldots$ have not, he eats his favorite house among $x, y, z \ldots$ at the speed $\omega_{i}(t)$.

Theorem 1 (Bogomolnaia and Moulin 2001) For each profile of eating speeds, the eating algorithm gives an ordinally efficient random assignment. Conversely, each ordinally efficient random assignment can be obtained by the eating algorithm for some profile of eating speeds.

The probabilistic serial (PS) solution is obtained by choosing uniform eating speeds: for each agent $i$, and for $0 \leq t \leq 1, \omega_{i}(t)=1$. The PS solution satisfies no envy but it is not strategy-proof.

The eating algorithm does not distinguish between existing tenants and new applicants. Before it determines the consumption of existing tenant $i$, it may have already allocated all the houses in $U_{i}$. Thus, in general, the assignment given by the eating algorithm is not individually rational. An extension of the eating algorithm to account for individual rationality is the eating rate (ER) algorithm (Sethuraman 2001). The ER algorithm is designed to adjust the eating speeds of the existing tenants so that each existing tenant's house is secured. Very briefly, whenever existing tenant $i$ 's house is demanded more than one agent, the eating speed of agent $i$ is increased to secure his endowment. For the details, we provide the complete description.

The eating rate (ER) algorithm (Sethuraman 2001). Let $\omega=\left(\omega_{i}\right)_{i \in I}$ be a profile of eating speeds. Given $\omega$, each agent $i$ is given an 'eating rate' $s_{i}(\cdot)$, and at time $t$, 
agent $i$ eats his most preferred house among all the houses still available at time $t$, at the rate $s_{i}(t)$. Let $e_{i}(t)$ be the amount that agent $i$ has eaten by the time $t$. At time $t$, agent $i$ needs $1-e_{i}(t)$ units of houses. If, at time $t$, the available portion of the house $\mu^{0}(i)$, that is the portion not yet eaten by any agent, exceeds what existing tenant $i$ needs, i.e. $1-e_{i}(t)$, then we say that the house $\mu^{0}(i)$ is publicly available at time $t$. At time $t$, the eating rate profile $s(t)$ is determined as follows: each new applicant eats at the rate $\omega_{i}(t)$. If the house $\mu^{0}(i)$ is publicly available at time $t$, then existing tenant $i$ eats at the rate $\omega_{i}(t)$. If the house $\mu^{0}(i)$ is not publicly available at time $t$, then the eating rate of agent $i$ depends on the sum of the eating rates of the agents, who eat $\mu^{0}(i)$ at time $t$. If this sum is less than or equal to $\omega_{i}(t)$, then existing tenant $i$ eats at the rate $\omega_{i}(t)$; otherwise, the eating rate of existing tenant $i$ is equal to this sum. A key point to note is that whenever a cycle $\left(i_{1}, i_{2}, i_{3}, \ldots i_{k}\right)$ is formed among the existing tenants such that for $j=1, \ldots, k-1$, agent $j$ eats the house owned by agent $j+1$, and agent $k$ eats the house owned by agent 1 , and if no portion of any of these houses is publicly available, then each agent in the cycle is assigned the appropriate portion of the house of the next agent in the cycle (the same idea as in the top trading cycle solution discussed in the introduction). Specifically, the appropriate amount swapped will be the largest quantity that is no greater than the residual requirement of an agent, where the residual requirement of agent $i$ is defined as $1-e_{i}(t)$.

The uniform eating rate (UER) solution is obtained by choosing uniform eating speeds. The UER solution reduces to the TTC solution when there are no new applicants, and to the PS solution when there are no existing tenants. This solution is ordinally efficient and individually rational, but it is not weakly strategy-proof.

While the eating algorithm characterizes the set of ordinally efficient assignments (Theorem 1), the following example illustrates why it is not possible to obtain a similar result with the ER algorithm.

Example 1 Consider the following preferences: (A boxed house below stands for the private endowment.)

$$
\begin{array}{lll}
\frac{R_{1}}{h_{2}} & \frac{R_{2}}{h_{1}} & \frac{R_{3}}{h_{1}} \\
h_{3} & h_{3} & h_{2} \\
h_{1} & h_{2} & h_{3}
\end{array}
$$


In this example, since individual rationality imposes no constraints at all (namely, any allocation is individually rational), the natural extension of the eating algorithm should ignore individual rationality and give the same allocation as the eating algorithm. However, agents 1 and 2 form a cycle at time 0, and for each profile of eating speed, the ER algorithm allocates $h_{2}$ to agent 1 and $h_{1}$ to agent 2 . Thus, the ER algorithm does not characterize the set of individually rational and ordinally efficient assignments: Consider an individually rational and ordinally efficient assignment $Q$ other than the one obtained by the ER algorithm. Since the set of random assignments given by the family of the ER algorithms is a singleton, it is not possible to obtain the assignment $Q$ with the ER algorithm. Moreover, the fairness aspect of the PS solution is not present in the UER solution: the allocation is unfair to agent 3; while agents 1 and 2 are assigned their most preferred houses, agent 3 is assigned to the bottom house in his ranking, although this is not imposed by individual rationality. ${ }^{10}$

\section{The generalized eating algorithm}

We construct the generalized eating $(G E)$ algorithm, which is the natural generalization of the eating algorithm so as to account for individual rationality. The GE algorithm follows the general outline of the eating algorithm. As soon as the individual rationality (IR) constraint of a subset of existing tenants becomes binding, the algorithm pledges the remainder of the acceptable houses of these existing tenants -'bottleneck' groupto them. At this point, the bottleneck group and the remainder of their acceptable houses constitute a sub-problem; the rest of the agents and the remainder of the rest of the houses constitute another sub-problem. The GE algorithm proceeds in each sub-problem in a recursive manner. Other IR constraints may become binding within the sub-problem or sub-sub-problem etc. Whenever that happens the remainders of acceptable houses of the bottleneck group are pledged to the group. Within each problem, defined by a bottleneck group of agents and the remainders of their acceptable houses, the GE algorithm proceeds with the eating algorithm until possibly another bottleneck group appears, and so on. It continues until all houses are allocated.

Let $r_{h}(t)$ be the remainder of house $h$ at time $t$. At time 0 , there is one unit of each house available. What remains of a set of houses $H^{\prime} \subseteq H$ is the sum of what remains

\footnotetext{
${ }^{10}$ For the formal definition of fairness, see Section 5.1.
} 
of each house in that set: for each time $t$,

$$
r_{H^{\prime}}(t)=\sum_{h \in H^{\prime}} r_{h}(t)
$$

At time $t$, agent $i$ has eaten $\int_{0}^{t} \omega_{i}(y) d y$ units and needs $1-\int_{0}^{t} \omega_{i}(y) d y$ units more to obtain a (random) consumption. Thus, for each nonempty subset $S$ of $I_{E}$, the slack of the IR constraint of $S$ at time $t$, denoted by $r_{d_{S}}(t),{ }^{11}$ is the difference between the remainder of $U_{S}$ and the total amount that the agents in $S$ need:

$$
r_{d_{S}}(t)=r_{U_{S}}(t)-\sum_{i \in S}\left(1-\int_{0}^{t} \omega_{i}(y) d y\right)
$$

A bottleneck group appears in the algorithm when the slack of the associated IR constraint is equal to zero. These critical points in time are determined by the following argument: First, note that each agent in $S$ eats a house in $U_{S}$ at time $t$. As long as only the agents in $S$ eat a house in $U_{S}$ at time $t$, the slack, $r_{d_{S}}(t)$, remains constant. However, if there are other agents eating a house in $U_{S}$, then the slack decreases at a rate equal to the aggregate eating speed of these agents. For each nonempty subset $S$ of $I_{E}$, let $\omega_{d_{S}}(t)$ be the aggregate eating speed of the agents, each of whom belongs to $I \backslash S$ and eats a house in $U_{S}$ at time $t$. Thus, the slack at time $t, r_{d_{S}}(t)$, decreases at the rate $\omega_{d_{S}}(t)$. By keeping track of each slack and the rate at which each slack decreases, we determine when a subset of existing tenants becomes a bottleneck group.

The generalized eating (GE) algorithm. Initialization: Let $t=0, N \equiv I$, and the remainder of each house in $H$ be 1 . Recursive step: The algorithm starts at time $t$. Each agent $i$ eats his favorite available house at the speed of $\omega_{i}(t)$ and for each $S \subseteq I_{E} \cap N$ (if $N \subseteq I_{E}$, then for each $S \varsubsetneqq N$ ), the slack $r_{d_{S}}(t)$ decreases at the rate $\omega_{d_{S}}(t)$. It continues until the IR constraint of, say $S_{0}$, is binding (i.e. until the slack $r_{d_{S_{0}}}$ is equal to zero). The set $S_{0}$ is the bottleneck group. (If there is more than one bottleneck group, the algorithm arbitrarily chooses one of them.) At this time, say $t_{0}$, the recursive step is applied to the following two sub-problems. The first sub-problem:

\footnotetext{
${ }^{11}$ Note that $r$ denotes both the remainder of a house and also the slack of an IR constraint. We use the subscript $d_{S}$ for the IR constraint of $S$ to distinguish it from houses. One can consider the slack of an IR constraint, say $r_{d_{S}}$, as the remainder of a dummy house $d_{S}$, being eaten at a certain speed.
} 
Let $t=t_{0}, N \equiv S_{0}$, and the remainder of each house $h$ be $r_{h}\left(t_{0}\right)$. The second subproblem: Let $t=t_{0}, N \equiv I \backslash S_{0}$, the remainder of each house $h$ in $H \backslash U_{S_{0}}$ be $r_{h}\left(t_{0}\right)$, and the remainder of each house $h$ in $U_{S_{0}}$ be 0 .

Example 2 Consider the profile of uniform eating speeds: for each $t, 0 \leq t \leq 1$, and for each $i \in S, \omega_{i}(t)=1$. Note that, in this case,

$$
r_{d_{S}}(t)=r_{U_{S}}(t)-(1-t)|S|
$$

and $\omega_{d_{S}}(t)$ is the number of agents, each of whom belongs to $I \backslash S$ and eats a house in $U_{S}$ at time $t$. Consider the following preferences:

$$
\begin{array}{cccccc}
\frac{R_{1}}{h_{2}} & \frac{R_{2}}{h_{3}} & \frac{R_{3}}{h_{1}} & \frac{R_{4}}{h_{2}} & \frac{R_{5}}{h_{1}} & \frac{R_{6}}{h_{3}} \\
h_{3} & h_{2} & h_{4} & h_{4} & h_{4} & h_{2} \\
h_{1} & & h_{3} & h_{5} & h_{3} & h_{5} \\
& & & h_{6} & h_{6} & h_{4} \\
& & h_{1} & h_{2} & h_{6} \\
& & & h_{3} & h_{5} & h_{1}
\end{array}
$$

Let $Q$ denote the random assignment generated by the generalized eating algorithm.

Step 1: We identify each IR constraint, the associated slack and the rate at which it decreases at time zero. For convenience, for each $\{a, b, c, \ldots\} \subseteq I_{E}$, let $d_{a b c .}$ denote $d_{\{a, b, c, \ldots\}}$. Thus, there are seven constraints: $d_{1}, d_{2}, d_{3}, d_{12}, d_{13}, d_{23}, d_{123}$

$\begin{array}{ccccc} & \left|U_{S}\right| & |S| & r_{d_{S}}(0) & \omega_{d_{S}}(0) \\ d_{1} & 3 & 1 & 2 & 5 \\ d_{2} & 2 & 1 & 1 & 3 \\ d_{3} & 3 & 1 & 2 & 3 \\ d_{12} & 3 & 2 & 1 & 4 \\ d_{13} & 4 & 2 & 2 & 4 \\ d_{23} & 4 & 2 & 2 & 4 \\ d_{123} & 4 & 3 & 1 & 3\end{array}$

Each agent eats his favorite available house and each slack $r_{d_{S}}$ decreases at the rate $\omega_{d_{S}}$. The bottleneck set is $\{1,2\}$; at time $t^{1}=\frac{1}{4}, r_{d_{12}}(t)$ is equal to zero. At this time, 
none of the houses is fully allocated; the partial assignment is as follows: $q_{12}=q_{23}=$ $q_{31}=q_{42}=q_{51}=q_{63}=\frac{1}{4}$.

Step 2: Sub-problem $\{1,2\}$. The remainder of $U_{\{1,2\}}=U_{1} \cup U_{2}$, i.e. half of $h_{1}, h_{2}$ and $h_{3}$, will be allocated to agents 1 and 2 .

$$
\text { IR constraints: }\left\{\begin{array}{ccccc} 
& \sum_{h \in U_{S}} r_{h}\left(t^{1}\right) & \left(1-t^{1}\right)|S| & r_{d_{S}}\left(t^{1}\right) & \omega_{d_{S}}\left(t^{1}\right) \\
d_{1} & \frac{3}{2} & \frac{3}{4} & \frac{3}{4} & 1 \\
d_{2} & 1 & \frac{3}{4} & \frac{1}{4} & 1
\end{array}\right.
$$

Agent 1 eats $h_{2}$ and agent 2 eats $h_{3}$ until the slack of the IR constraint of agent 2 is zero, which occurs at time $t^{1}+t^{\prime}=\frac{1}{4}+\frac{1}{4}$. At this time, partial assignment is as follows: $q_{12}^{\prime}=q_{23}^{\prime}=\frac{1}{4}$.

Step 3: Sub-sub-problem $\{2\}$. The remainder of $U_{2}$, i.e. a quarter of $h_{2}$ and a quarter of $h_{3}$, will be allocated to agent 2 , and the rest to agent 1 . The assignment is as follows: $Q_{11}\left(\{1,2\}, t^{1}\right)=Q_{23}\left(\{1,2\}, t^{1}\right)=\frac{1}{2} ; Q_{12}\left(\{1,2\}, t^{1}\right)=Q_{22}\left(\{1,2\}, t^{1}\right)=\frac{1}{4}$. Each house $h$ is allocated and for $k=1,2$, agent $k$ is assigned $\frac{3}{4}=\left(1-t^{1}\right)|\{k\}|$ units. Agents 1 and 2 leave. The partial assignment is as follows: $q_{11}=\frac{1}{2}, q_{12}=\frac{1}{2}, q_{22}=\frac{1}{4}, q_{23}=\frac{3}{4}$, $q_{31}=q_{42}=q_{51}=q_{63}=\frac{1}{4}$.

Step 4: The remaining agents are 3, 4, 5, 6 and remaining houses are $h_{4}, h_{5}, h_{6}$. The only remaining existing tenant is agent 3 .

$$
\begin{array}{ccccc} 
& \sum_{h \in U_{S}} r_{h}\left(t^{1}\right) & \left(1-t^{1}\right)|\{3\}| & r_{d_{3}}\left(t^{1}\right) & \omega_{d_{3}}\left(t^{1}\right) \\
d_{3} & 1 & \frac{3}{4} & \frac{1}{4} & 2
\end{array}
$$

At time $t^{2}=t^{1}+\frac{r_{d_{3}}\left(t^{1}\right)}{\omega_{d_{3}}\left(t^{1}\right)}=\frac{3}{8}$, the slack $r_{d_{3}}\left(t^{2}\right)$ is equal to zero. At this time, the partial assignment is as follows: $q_{31}=q_{42}=q_{51}=q_{63}=\frac{1}{4}$ and $q_{34}=q_{44}=q_{54}=q_{65}=\frac{1}{8}$.

Step 5: Sub-problem $\{3\}$. The remainder of $h_{4}$ will be allocated to agent 3. Thus, $Q_{34}\left(\{3\}, t^{2}\right)=\frac{5}{8}$. Agent 3 leaves: $q_{31}=\frac{1}{4}, q_{34}=\frac{1}{8}+\frac{5}{8}=\frac{3}{4}$.

Step 6: Since each remaining agent is a new applicant, there are no bottleneck sets in 
the rest of the algorithm. The random assignment is as follows:

$Q=\begin{array}{ccccccc} & h_{1} & h_{2} & h_{3} & h_{4} & h_{5} & h_{6} \\ 1 & \frac{1}{2} & \frac{1}{2} & 0 & 0 & 0 & 0 \\ 2 & 0 & \frac{1}{4} & \frac{3}{4} & 0 & 0 & 0 \\ 3 & \frac{1}{4} & 0 & 0 & \frac{3}{4} & 0 & 0 \\ 4 & 0 & \frac{1}{4} & 0 & \frac{1}{8} & \frac{7}{16} & \frac{3}{16} \\ 5 & \frac{1}{4} & 0 & 0 & \frac{1}{8} & 0 & \frac{10}{16} \\ 6 & 0 & 0 & \frac{1}{4} & 0 & \frac{9}{16} & \frac{3}{16}\end{array}$

We turn to the formal definition of the generalized eating algorithms. We use the following notation: whenever $h \in H^{\prime}$, let

$$
M\left(h, H^{\prime}\right)=\left\{i \in I: h P_{i} h^{\prime} \forall h^{\prime} \in H^{\prime}, h^{\prime} \neq h\right\}
$$

and $m\left(h, H^{\prime}\right)=\left|M\left(h, H^{\prime}\right)\right|$. Given an ordinal preference profile $R=\left(R_{i}\right)_{i \in I} \in \mathcal{D}^{|I|}$ and an endowment profile $\mu^{0} \in \mathcal{M}^{0}$, the random assignment corresponding to the profile $\omega$ is denoted by $Q(\omega)$ and defined by the following recursive procedure.

Let $I_{E}^{0}=I, H^{0}=H, t^{0}=0$, and $Q^{0}=[0]$ (the $|I| \times|I|$ matrix of zeros). For each $h \in H$, let $r_{h}(0)=1$. For each $\emptyset \neq S \subseteq I_{E}$, let $r_{d_{S}}(0)=\left|U_{S}\right|-|S|$ and for $t \geq 0$,

$$
\omega_{d_{S}}(t)=\sum_{i \in\left(\bigcup_{h \in U_{S}} M(h, H)\right) \backslash S} \omega_{i}(t) .
$$

Let $k \in \mathbb{N}$, and suppose $I_{E}^{k-1}, H^{k-1}, t^{k-1}, Q^{k-1}, r\left(t^{k-1}\right), \omega\left(t^{k-1}\right)$ have been defined. For each $h \in H^{k-1}$, define

$$
\begin{aligned}
t^{k}(h) & =\min \left\{t \mid \sum_{i \in M\left(h, H^{k-1}\right)_{t^{k-1}}} \int_{i}^{t} \omega_{i}(y) d y=r_{h}\left(t^{k-1}\right)\right\} \\
\left(t^{k}(h)\right. & \left.=+\infty, \text { if } M\left(h, H^{k-1}\right)=\emptyset\right) .
\end{aligned}
$$


Let

$$
\begin{aligned}
& t^{k}=\min \left\{\min _{h \in H^{k-1}} t^{k}(h), \min _{S \in I_{E}^{k-1}}\left\{t(S) \mid r_{d_{S}}\left(t^{k-1}\right)=\int_{t^{k-1}}^{t(S)} \omega_{d_{S}}(y) d y\right\}\right\} \\
& B^{k} \equiv\left\{S \subseteq I_{E}^{k-1}: t^{k}=t(S)\right\} \\
& I_{E}^{k} \equiv I_{E}^{k-1} \backslash\left(\bigcup_{S \in B^{k}} S\right) \\
& H^{k} \equiv H^{k-1} \backslash\left(\left(\bigcup_{S \in B^{k}} U_{S}\right) \bigcup\left\{h: t^{k}=t^{k}(h)\right\}\right) .
\end{aligned}
$$

For each $h \in H^{k}$, let

$$
r_{h}\left(t^{k}\right)=r_{h}\left(t^{k-1}\right)-\sum_{i \in M\left(h, H^{k-1}\right.} \int_{t^{k-1}}^{t^{k}} \omega_{i}(y) d y
$$

For each $S \subseteq I_{E}^{k}$, let

$$
r_{d_{S}}\left(t^{k}\right)=r_{U_{S}}\left(t^{k}\right)-\sum_{i \in S}\left(1-\int_{0}^{t^{k}} \omega_{i}(y) d y\right)
$$

and for $t \geq t^{k}$,

$$
\omega_{d_{S}}\left(t^{k}\right)=\sum_{i \in\left(\bigcup_{h \in H^{k} \cap U_{S}} M\left(h, H^{k}\right)\right) \backslash S} \omega_{i}(t) .
$$

We call each $S \in B^{k}$ a bottleneck set. Let $Q(\omega)\left(\bigcup_{S \in B^{k}} S, t^{k}\right)$ denote the assignment of the houses in $\left(H^{k-1} \backslash\left\{h: t^{k}=t^{k}(h)\right\}\right) \cap U \cup_{S \in B^{k}} S$ to the agents in $\bigcup_{S \in B^{k}} S$, given by the generalized eating algorithm, where the remainder of house $h$ is $r_{h}\left(t^{k}\right)$, and each agent 
$i \in \bigcup_{S \in B^{k}} S$ receives exactly $\left(1-\int_{0}^{t^{k}} \omega_{i}(y) d y\right)$ units. Define

$$
Q^{k}: q_{i h}^{k}=\left\{\begin{array}{rr}
q_{i h}^{k-1} & \text { if } i \notin M\left(h, H^{k-1}\right) \\
\text { and } i \notin \bigcup_{S \in B^{k}} S \\
q_{i h}^{k-1}+t^{k}-t^{k-1} & \text { if } i \in M\left(h, H^{k-1}\right) \\
q_{i h}^{k-1}+q(\omega)_{i h}\left(\bigcup_{S \in B^{k}} S, t^{k}\right) & \text { and } i \notin \bigcup_{S \in B^{k}} S \\
q_{i h}^{k-1}+t^{k}-t^{k-1}+q(\omega)_{i h}\left(\bigcup_{S \in B^{k}} S, t^{k}\right) & \text { if } i \notin M\left(h, H^{k-1}\right) \\
\text { and } i \in \bigcup_{S \in B^{k}} S & \text { and } i \in M\left(h, H^{k-1}\right) \\
\bigcup_{S \in B^{k}} S
\end{array}\right.
$$

The random assignment $Q(\omega)$ is the one obtained by the above recursive procedure: $Q(\omega)=Q^{K}$ where all the houses are allocated at $t^{K}$.

If, for some $S \subseteq I_{E}, r_{d_{S}}(t)=0$, then the constraint is binding and individual rationality implies that the remainder of $U_{S}$ has to be allocated only to the agents in $S$. Thus, a necessary condition for individual rationality is that the slack of the IR constraint of each subset of existing tenants is nonnegative. It turns out that this is also sufficient (Lemma 1): At time $t$, if, for each $T \subseteq S, r_{d_{T}}(t) \geq 0$, then there is an allocation of the remainder of $U_{S}$, such that the random consumption of each agent in $S$ is individually rational for him. This is a direct consequence of the following result: ${ }^{12}$

Theorem 2 (The Supply-Demand Theorem, Gale 1957). Assume that there are $k$ suppliers $h_{1}, h_{2}, \ldots, h_{k}$ of some commodity and $n$ consumers $i_{1}, i_{2}, \ldots, i_{n}$. Let $U_{i}$ be the set of suppliers who can ship to consumer $i$. Let $r_{h}$ be the supply of supplier of $h$, and $\lambda(i)$ be the demand of consumer $i$, and $x_{a b}$ denote the shipment from supplier $h_{a}$ to consumer $i_{b}$. Then, there is a shipment vector $x=\left(x_{a b}\right)_{a, b}$ such that

$$
\text { for each } a \in\{1, \ldots, k\}: \sum_{b} x_{a b} \leq r_{h_{a}}
$$

\footnotetext{
${ }^{12}$ Gale's Theorem is a generalization of Hall's Set Representation Theorem (1935) which holds only for integers.
} 


$$
\begin{gathered}
\text { for each } b \in\{1, \ldots, n\}: \sum_{a} x_{a b} \geq \lambda\left(i_{b}\right), \\
x_{a b}>0 \text { implies } h_{a} \in U_{i_{b}}
\end{gathered}
$$

if, and only if, for each subset $S$ of consumers

$$
\sum_{i \in S} \lambda(i) \leq \sum_{h \in \cup_{i \in S} U_{i}} r_{h}
$$

The following lemma is a corollary to this theorem:

Lemma 1 Let $S$ be a subset of existing tenants. At time $t$, the remainder of house $h$ is $r_{h}(t)$ and each agent $i$ in $S$ needs $1-\int_{0}^{t} \omega_{i}(y) d y$ units from the houses in $U_{i}$. Then, each agent in $S$ can be satisfied if, and only if, for each $T \subseteq S$,

$$
\sum_{h \in U_{T}} r_{h}(t) \geq \sum_{i \in T}\left(1-\int_{0}^{t} \omega_{i}(y) d y\right) .
$$

The GE algorithm is designed so as to maintain the nonnegativity of each slack at each time, and Lemma 1 says that the nonnegativity of each slack is sufficient to satisfy each existing tenant. This is the argument behind the following result.

Theorem 3 Let $R=\left(R_{i}\right)_{i \in I} \in \mathcal{D}^{|I|}$ be an ordinal preference profile and $\mu^{0} \in \mathcal{M}^{0}$ be an endowment profile. (1) For each profile of eating speed function $\omega=\left(\omega_{i}\right)_{i \in I}$, the random assignment $Q(\omega)$ is individually rational and ordinally efficient. (2) Conversely, for each individually rational and ordinally efficient random assignment $Q$ at $\left(\mu^{0}, R\right)$, there exists a profile $\omega$ such that $Q=Q(\omega)$.

\section{The individually rational probabilistic serial as- signment}

Definition 1 Given an endowment profile $\mu^{0} \in \mathcal{M}^{0}$ and an ordinal preference profile $R=\left(R_{i}\right)_{i \in I} \in \mathcal{D}^{|I|}$, the individually rational probabilistic serial assignment is the random assignment corresponding to the profile of uniform eating speeds: for each $t$, $0 \leq t \leq 1$, and for each $i \in S, \omega_{i}(t)=1$. It is denoted by $P S^{\mathbf{I R}}\left(\mu^{0}, R\right)$. 


\subsection{Fairness: No justified-envy}

When there are private endowments, individual rationality is an indispensable property for a solution. Unfortunately, individual rationality is incompatible with no envy. However, this does not mean that we should disregard fairness. Our approach is to weaken no envy by insisting that, while evaluating the equity of a solution, one should not ignore individual rationality. We argue that because of individual rationality, some violations of no-envy are justified.

Example 3 Illustration of unjustified envy. Consider the following preferences:

$$
\begin{array}{lll}
\frac{R_{1}}{h_{2}} & \frac{R_{2}}{h_{1}} & \frac{R_{3}}{h_{1}} \\
h_{1} & h_{2} & h_{2} \\
\hline h_{3} & h_{3} & h_{3}
\end{array}
$$

Individual rationality implies that house $h_{3}$ has to be allocated to agent 3. By ignoring this fact, one can conclude that such an allocation is not fair to agent 3 . On the other hand, if one accepts individual rationality as an indispensable requirement, one cannot deem it unfair.

Example 4 Illustration of justified envy. Consider the preferences and the endowment profile in Example 1. Consider an allocation at which agent 3 is assigned house $h_{3}$. Here, individual rationality imposes no constraints. Thus, agent 3 envies both agents 1 and 2. Even if one accepts individual rationality, the unfairness of this allocation is not justified.

Definition 2 i) A solution $\varphi: \mathcal{M}^{0} \times \mathcal{D}^{|I|} \rightarrow \mathcal{Q}$ satisfies no justified-envy if, for each $\mu^{0} \in \mathcal{M}^{0}$, each $R=\left(R_{i}\right)_{i \in I} \in \mathcal{D}^{|I|}$, and each $i, j \in I$ : either

$$
\varphi_{i}\left(\mu^{0}, R\right) s d\left(R_{i}\right) \varphi_{j}\left(\mu^{0}, R\right)
$$

or

$$
\operatorname{Support}\left(\varphi_{i}\left(\mu^{0}, R\right)\right) \backslash U_{j} \neq \emptyset .
$$

ii) A solution $\varphi: \mathcal{M}^{0} \times \mathcal{D}^{|I|} \rightarrow \mathcal{Q}$ satisfies weak no justified-envy if, for each 
$\mu^{0} \in \mathcal{M}^{0}$, each $R=\left(R_{i}\right)_{i \in I} \in \mathcal{D}^{|I|}$, and each $i, j \in I$ : either

$$
\varphi_{j}\left(\mu^{0}, R\right) s d\left(R_{i}\right) \varphi_{i}\left(\mu^{0}, R\right) \Rightarrow \varphi_{i}\left(\mu^{0}, R\right)=\varphi_{j}\left(\mu^{0}, R\right)
$$

or

$$
\operatorname{Support}\left(\varphi_{i}\left(\mu^{0}, R\right)\right) \backslash U_{j} \neq \emptyset \text {. }
$$

The PS ${ }^{\text {IR }}$ solution satisfies no justified-envy. The intuition for this result is as follows: At a given time, each agent eats his favorite available house. Also, a set of houses is eaten exclusively by a set of existing tenants only if otherwise, individual rationality is violated. The $\mathrm{PS}^{\mathbf{I R}}$ solution ignores the endowment profile as long as there is an allocation of the remainder of the available houses such that the resulting random assignment is individually rational. Thus, the existing tenants do not receive special treatment until egalitarianism can not be maintained without violating individual rationality. This is the reason for its fairness.

Proposition 1 The PS $S^{\mathbf{I R}}$ solution satisfies no justified-envy.

\section{$5.2 \quad$ Strategic Manipulation}

While the PS solution is weakly strategy-proof, the $\mathrm{PS}^{\mathbf{I R}}$ solution is not.

Example 5 The $P S^{\mathbf{I R}}$ solution is not weakly strategy-proof. Consider the following preferences:

$$
\begin{array}{lll}
\frac{R_{1}}{h_{2}} & \frac{R_{2}}{h_{1}} & \frac{R_{3}}{h_{2}} \\
h_{3} & h_{2} & h_{1} \\
h_{1} & h_{3} & h_{3}
\end{array}
$$

The PS ${ }^{\mathbf{I R}}$ solution gives the following probabilistic assignment:

$$
\operatorname{PS}^{\mathbf{I R}}\left(\mu^{0}, R\right)=\begin{array}{cccc} 
& h_{1} & h_{2} & h_{3} \\
1 & 0 & \frac{1}{2} & \frac{1}{2} \\
2 & 1 & 0 & 0 \\
3 & 0 & \frac{1}{2} & \frac{1}{2}
\end{array}
$$

Suppose agent 1 announces that he prefers $h_{2}$ to $h_{1}$ and $h_{1}$ to $h_{3}$. Let $R_{1}^{\prime}$ denote these preferences. The other agents are truthful. For these preferences, the PS ${ }^{\mathbf{I R}}$ solution 
assigns $h_{2}$ to agent 1 with probability 1 . Thus, $\operatorname{PS}_{1}^{\mathbf{I R}}\left(\mu^{0}, R_{1}^{\prime}, R_{-1}\right) s d\left(R_{1}\right) \operatorname{PS}_{1}^{\mathbf{I R}}\left(\mu^{0}, R\right)$.

In Example 5, agent 1 announces the reduced ranking of his true preferences on a proper subset of $U_{1}$. We call this type of misrepresentation a truncation (Roth and Rothblum 1999). Thus, the $\mathrm{PS}^{\mathbf{I R}}$ solution can be manipulated via truncation. The other possible manipulation (for the existing tenants or new applicants) is via reshuffling houses in the preference ranking and the PS ${ }^{\mathbf{I R}}$ solution can be manipulated via reshuffling as well (even in the weak sense).

Recently, Kojima and Manea (2007) showed that, in the PS solution, truthful reporting of ordinal preferences is a dominant strategy for an agent if the number of copies of each house type is sufficiently large. ${ }^{13}$ Given an agent and a set of houses, this result holds irrespective of the set of other agents and their ordinal preferences. However, when there are private endowments as well, this result does not extend for the $\mathrm{PS}^{\mathbf{I R}}$ solution: For any number of copies of the houses, say $h_{1}, h_{2}$ and $h_{3}$, it is possible to replicate the preferences $R_{1}, R_{1}^{\prime}, R_{2}$, and $R_{3}$ in Example 5 so that truncation makes agent 1 better off.

\subsection{Impossibility results}

The TTC solution from random orderings is strategy-proof but violates no justifiedenvy. The PS ${ }^{\mathbf{I R}}$ solution satisfies no justified-envy but not strategy-proofness. As the following theorem shows, the tension between these two properties is actually more severe.

Theorem 4 Assume $|I| \geq 3$. (i) No solution meets the following three requirements: individual rationality, strategy-proofness, and no justified-envy. (ii) No solution meets the following four requirements: individual rationality, ordinal efficiency, weak strategyproofness, and weak no justified-envy.

\subsection{An invariance property}

Individual rationality requires that each existing tenant finds his assignment at least as desirable as his endowment. However, there is no reason to accept that an existing tenant should have an advantage (or a disadvantage) besides this guarantee. Thus, we should distinguish the privilege of an existing tenant due to individual rationality from

\footnotetext{
${ }^{13}$ Note that, for the case of objective indifferences, the PS ${ }^{\mathbf{I R}}$ solution is unambiguously defined.
} 
other advantages (or disadvantages) related to his endowment: Suppose an existing tenant occupies a house, which is his worst house. Then, given that the number of houses is equal to the number of agents, any allocation is individually rational for him. In other words, the fact that he is an existing tenant is irrelevant in terms of individual rationality. Thus, a robust solution should be indifferent between whether an agent is the existing tenant of his worst house or he is a new applicant.

Definition 3 Given $\left(\mu^{0}, R\right) \in \mathcal{M}^{0} \times \mathcal{D}^{|I|}$, define $\mu^{1}\left(\mu^{0}, R\right) \in \mathcal{M}^{0}$ as follows:

$$
\mu^{1}(i)= \begin{cases}h_{0} & \text { if } i \in\left\{j \in I_{E}: U_{j}=H\right\} \\ \mu^{0}(i) & \text { otherwise }\end{cases}
$$

A solution $\varphi: \mathcal{M}^{0} \times \mathcal{D}^{|I|} \rightarrow \mathcal{Q}$ satisfies worst-object endowment invariance (WI) if, for each $\left(\mu^{0}, R\right) \in \mathcal{M}^{0} \times \mathcal{D}^{|I|}, \varphi\left(\mu^{0}, R\right)=\varphi\left(\mu^{1}\left(\mu^{0}, R\right), R\right)$.

Proposition 2 The PS $S^{\mathbf{I R}}$ solution satisfies worst-object endowment invariance.

\section{$6 \quad$ Extensions}

We list some extensions of our random assignment model. In all but one, the definition of the PS ${ }^{\mathbf{I R}}$ extends almost verbatim, and the properties are preserved.

\subsection{Different number of agents and houses, opting out}

Suppose there are $n$ agents and $m$ houses. If $n<m$, then a random assignment is a nonnegative and row-stochastic matrix; its rows sum to one and its columns sum to at most one. The algorithm remains the same and the results are preserved. If $n>m$, then a random assignment is a nonnegative and column-stochastic matrix; its rows corresponding to existing tenants sum to one, its rows corresponding to new applicants sum to $\frac{m-\left|I_{E}\right|}{n-\left|I_{E}\right|}$, and its columns sum to one. In this case, the characterization of ordinal efficiency in terms of the acyclicity of the relation $\tau$ (proof of Proposition 2) holds. The definition of the $\mathrm{PS}^{\mathbf{I R}}$ remains the same.

In some examples, an agent may prefer $h_{0}$ to some of the houses. The extension of the $\mathrm{PS}^{\mathbf{I R}}$ solution to this general case is straightforward: if an existing tenant prefers $h_{0}$ to his house, then he should be treated as a new applicant; otherwise, opting out is irrelevant for him. 


\subsection{Fractional endowments}

Suppose agents may own fractions of different houses. Let $e_{i}=\left(e_{i j}\right)_{j}$ be agent $i^{\prime}$ s endowment where $e_{i j}$ is agent $i^{\prime}$ s fractional endowment of house $h_{j} \in H$. Thus, an endowment profile can be represented by a sub-stochastic matrix:

$$
e=\begin{array}{ccccc} 
& h_{1} & h_{2} & \cdots & h_{n} \\
1 & e_{11} & e_{12} & \cdots & e_{1 n} \\
2 & e_{21} & e_{22} & \cdots & e_{2 n} \\
\vdots & \vdots & \vdots & \vdots & \vdots \\
n & e_{n 1} & e_{n 2} & \cdots & e_{n n}
\end{array}
$$

The PS ${ }^{\mathbf{I R}}$ solution extends to this general case as follows.

Let $e$ be an endowment profile as defined in the conclusion. A random assignment $Q$ is individually rational if, for each agent $i, Q_{i} s d\left(R_{i}\right) e_{i}$. For each positive fraction of a house endowed by an agent, individual rationality imposes a constraint. In order to keep track of these constraints, we define pseudo-agents associated with the fractions that the agents are endowed with. Let $I_{p}=\left\{(i, j): i \in I, e_{i j}>0\right\}$ be the set of pseudo-agents. The endowment of pseudo-agent $(i, j) \in I_{p}$ is $\mu^{0}(i, j)=\sum_{k: h_{k} \in U\left(R_{i}, h_{j}\right)} e_{i k}$. Let $\mathcal{S}$ be a family of subsets of $I_{p}$ such that $S \in \mathcal{S}$ if, and only if, for $\left(i_{1}, j_{1}\right),\left(i_{2}, j_{2}\right) \in S$, $i_{1}=i_{2}$ implies $j_{1}=j_{2}$.

The remainder of a house at time $t, r_{h}(t)$, is defined as before. For $S=\left\{\left(i_{1}, j_{1}\right)\right.$, $\left.\left(i_{2}, j_{2}\right), \ldots,\left(i_{k}, j_{k}\right)\right\} \in \mathcal{S}$, the slack of the IR constraint of $S$ at time $t$ is

$$
r_{d_{S}}(t)=r \underset{(i, j) \in S}{\bigcup} U\left(R_{i}, h_{j}\right)(t)-\sum_{(i, j) \in S} \mu^{0}(i, j)+t|S|
$$

Each agent $i \in I$ eats his favorite available house at the speed 1. For each $S \in \mathcal{S}$, let $\omega_{d_{S}}(t)$ be the number of agents, each of whom belongs to $I \backslash\{i:(i, j) \in S$ for some $j$ \} and eats a house in $\bigcup_{(i, j) \in S} U\left(R_{i}, h_{j}\right)$ at time $t$. Thus, the slack at time $t, r_{d_{S}}(t)$, decreases at the rate $\omega_{d_{S}}(t)$. By keeping track of each slack and the rate at which each slack decreases, we determine when a subset of pseudo-agents becomes a bottleneck group. Let $S_{0}$ be a bottleneck group at time $t_{0}$. As in the GE algorithm, at time $t_{0}$, we define two sub-problems and solve them recursively. 
The first sub-problem: Let $t=t_{0} ;\left\{i:(i, j) \in S_{0}\right.$ for some $\left.j\right\}$ be the set of agents, and

$$
N=\left\{(i, k) \text { : for some } j,(i, j) \in S_{0}, e_{i k}>0 \text { and } h_{k} \in U\left(R_{i}, h_{j}\right)\right\}
$$

be the set of pseudo-agents; and the remainder of each house $h$ be $r_{h}\left(t_{0}\right)$.

The second sub-problem: Let $t=0 ; I \backslash\left\{i:(i, j) \in S_{0}\right.$ and $\left.\mu^{0}(i, j)=\sum_{l} e_{i l}\right\}$ be the set of agents; $I_{p} \backslash N$ be the set of pseudo-agents; the remainder of each house $h$ in $H \backslash \bigcup_{(i, j) \in S_{0}} U\left(R_{i}, h_{j}\right)$ be $r_{h}\left(t_{0}\right)$, and the remainder of each house $h$ in $\bigcup_{(i, j) \in S_{0}} U\left(R_{i}, h_{j}\right)$ be 0 . Also, update the endowment profile to $e^{\prime}$ as follows: if $(i, k) \in N$, then let $e_{i k}^{\prime}=0$; otherwise, let $e_{i k}^{\prime}=e_{i k}$.

\subsection{Weak preferences}

We assumed that preferences are strict, and this assumption is critical for our results. However, there are both practical and technical reasons to consider the more general weak preferences domain, yet, the GE algorithm is not well-defined when agents are allowed to be indifferent between houses. The assignment problem with private endowments and a social endowment on the weak preferences domain has been recently studied (Yllmaz, forthcoming), and the connection between this specific assignment problem and the parametric network flow problem is established. The non-triviality of this connection is due to the individual rationality constraints.

\section{Conclusion}

We proposed the natural generalization of the eating algorithm to account for individual rationality. The generalized eating algorithm characterizes the set of individually rational and ordinally efficient random assignments. Also, a special solution in the class of solutions reduced by the family of generalized eating algorithms achieves a new fairness axiom (no justified envy); however, it does not satisfy strategy-proofness, even in the weak sense. We also show that no justified envy, strategy-proofness and individual rationality are incompatible.

While there are many real-life resource allocation problems that correspond to our model, here we present two of them: school choice and kidney exchange problems.

In a school choice problem, there are a number of students each of whom should 
be assigned a seat at one of a number of schools (Abdulkadiroğlu and Sönmez 2003). Each student has strict preferences over all schools, and each school has a strict priority ordering of all students. Here, priorities are imposed by state or local laws. Several central assignment mechanisms are adopted to place students to schools, and it is not rare that some students are not assigned to any of the schools by these mechanisms in the first round. For example, in New York City, the number of students who are unassigned after the main round is over 8,000 students (Pathak 2006). However, each student has the right to attend school, and to place the unassigned students, a supplementary round is organized. In this round, students are asked to submit a new ranking of the participant schools, and no school ranks the students. Our model corresponds to this assignment problem when, along unassigned students, there are also some existing students who wish to transfer their assignments.

A kidney exchange problem consists of incompatible patient-donor pairs; the kidney of the donor cannot be transplanted to the patient (the intended recipient) due to medical incompatibilities (Roth et al. 2004). Each patient has a preference ordering over the set of donors. In our language, the patients are the existing tenants, and the donors are the occupied houses. Individual rationality implies that each patient either receives a compatible donor kidney transplantation or he remains with his donor. No justified-envy implies that for each $i, j \in I$ either patient $i$ does not envy patient $j$ or at least one of the donors that patient $i$ is assigned with positive probability is not compatible with patient $j$. The PS ${ }^{\text {IR }}$ is an alternative solution satisfying these properties and ordinal efficiency.

\section{Appendix: Proofs}

\section{PROOF OF THEOREM 3}

Proof of Part 1: Let $R=\left(R_{i}\right)_{i \in I} \in \mathcal{D}^{|I|}$ be an ordinal preference profile and $\mu^{0} \in \mathcal{M}^{0}$ be an endowment profile. Let $\omega=\left(\omega_{i}\right)_{i \in I}$ be a profile of eating speeds. In order to show that the random assignment $Q(\omega)=\left[q(\omega)_{i h}\right]_{i \in I, h \in H}$ is individually rational and ordinally efficient, we need two more lemmas in addition to Lemma 1 in Section 4.

Lemma 2 Let $t^{k} \in[0,1]$ such that $B^{k} \neq \emptyset$. If $S_{1}, S_{2} \in B^{k}$, then

i) $S_{1} \cap S_{2} \in B^{k}$,

ii) $S_{1} \cup S_{2} \in B^{k}$, 
iii) $S_{1} \cap S_{2}=\emptyset \Rightarrow\left(H^{k-1} \backslash\left\{h: t^{k}=t^{k}(h)\right\}\right) \cap\left(U_{S_{1}} \cap U_{S_{2}}\right)=\emptyset$.

Proof Let $S_{1}, S_{2} \in B^{k}$, and $H^{\prime}=\left(U_{S_{1} \backslash S_{2}} \cap U_{S_{2} \backslash S_{1}}\right) \backslash U_{S_{1} \cap S_{2}}$. By definition of a bottleneck set, for $S=S_{1}, S_{2}, r_{d_{S}}\left(t^{k}\right)=0$, thus,

$$
\sum_{h \in H^{k-1} \cap U_{S}}\left(r_{h}\left(t^{k-1}\right)-\sum_{i \in M\left(h, H^{k-1}\right.} \int_{t^{k-1}}^{t^{k}} \omega_{i}(y) d y\right)=\sum_{i \in S}\left(1-\int_{0}^{t^{k}} \omega_{i}(y) d y\right) .
$$

By adding these two equalities for $S_{1}$ and $S_{2}$,

$$
\begin{aligned}
& \sum_{h \in H^{k-1} \cap H^{\prime}}\left(r_{h}\left(t^{k-1}\right)-\sum_{i \in M\left(h, H^{k-1}\right)_{t^{k-1}}} \int_{i}^{t^{k}} \omega_{i}(y) d y\right) \\
& +\sum_{h \in H^{k-1} \cap U_{S_{1} \cap S_{2}}}\left(r_{h}\left(t^{k-1}\right)-\sum_{i \in M\left(h, H^{k-1}\right.} \int_{t^{k-1}}^{t^{k}} \omega_{i}(y) d y\right)-\sum_{i \in S_{1} \cap S_{2}}\left(1-\int_{0}^{t^{k}} \omega_{i}(y) d y\right) \\
& +\sum_{h \in H^{k-1} \cap U_{S_{1} \cup S_{2}}}\left(r_{h}\left(t^{k-1}\right)-\sum_{i \in M\left(h, H^{k-1}\right.} \int_{t^{k-1}}^{t^{k}} \omega_{i}(y) d y\right)-\sum_{i \in S_{1} \cup S_{2}}\left(1-\int_{0}^{t^{k}} \omega_{i}(y) d y\right) \\
& =0 .
\end{aligned}
$$

First note that, by definition of $t^{k}$,

$$
\sum_{h \in H^{k-1} \cap H^{\prime}}\left(r_{h}\left(t^{k-1}\right)-\sum_{i \in M\left(h, H^{k-1}\right)_{t^{k-1}}} \int_{t^{k}}^{t^{k}} \omega_{i}(y) d y\right) \geq 0
$$

If $S_{1} \cap S_{2}$ is not a bottleneck set, then

$$
\sum_{h \in H^{k-1} \cap U_{S_{1} \cap S_{2}}}\left(r_{h}\left(t^{k-1}\right)-\sum_{i \in M\left(h, H^{k-1}\right.} \int_{t^{k-1}}^{t^{k}} \omega_{i}(y) d y\right)>\sum_{i \in S_{1} \cap S_{2}}\left(1-\int_{0}^{t^{k}} \omega_{i}(y) d y\right),
$$

which implies

$$
\sum_{h \in H^{k-1} \cap U_{S_{1} \cup S_{2}}}\left(r_{h}\left(t^{k-1}\right)-\sum_{i \in M\left(h, H^{k-1}\right.} \int_{t^{k-1}}^{t^{k}} \omega_{i}(y) d y\right)<\sum_{i \in S_{1} \cup S_{2}}\left(1-\int_{0}^{t^{k}} \omega_{i}(y) d y\right) .
$$


This means that the slack of the IR constraint of $S_{1} \cup S_{2}$ is negative at time $t^{k}$, and it is equal to zero before $t^{k}$, thus

$$
r_{d_{S_{1} \cup S_{2}}}\left(t^{k-1}\right)=\int_{t^{k-1}}^{t\left(S_{1} \cup S_{2}\right)} \omega_{d_{S_{1} \cup S_{2}}}(y) d y, \quad \text { where } t\left(S_{1} \cup S_{2}\right)<t^{k}
$$

But this contradicts the definition of $t^{k}$. Thus, $S_{1} \cap S_{2} \in B^{k}$. By the same argument, $S_{1} \cup S_{2} \in B^{k}$ as well. Moreover, these imply

$$
\sum_{h \in H^{k-1} \cap H^{\prime}}\left(r_{h}\left(t^{k-1}\right)-\sum_{i \in M\left(h, H^{k-1}\right.} \int_{t^{k-1}}^{t^{k}} \omega_{i}(y) d y\right)=0 .
$$

Also, if $S_{1} \cap S_{2}=\emptyset$, then $H^{\prime}=U_{S_{1}} \cap U_{S_{2}}$. Thus, by equality (1), $h \in H^{k-1} \cap\left(U_{S_{1}} \cap U_{S_{2}}\right)$ implies $r_{h}\left(t^{k-1}\right)-\sum_{i \in M\left(h, H^{k-1}\right)} \int_{t^{k-1}}^{t^{k}} \omega_{i}(y) d y=0$, which means $t^{k}(h)=t^{k}$. QED

Lemma 3 For each $k, S \subseteq I_{E}^{k}$ implies $r_{d_{S}}\left(t^{k}\right)>0$.

Proof If $B^{k}=\emptyset$, the result follows immediately. Let $S^{\prime}=\underset{S_{j} \in B^{k}}{\cup} S_{j}$ be nonempty. Suppose there is $S^{\prime \prime} \subseteq I_{E}^{k}$ such that

$$
r_{d_{S^{\prime \prime}}}\left(t^{k}\right)=\sum_{h \in H^{k} \cap U_{S^{\prime \prime}}} r_{h}\left(t^{k}\right)-\sum_{i \in S^{\prime \prime}}\left(1-\int_{0}^{t^{k}} \omega_{i}(y) d y\right) \leq 0
$$

Since houses in $U_{S^{\prime}}$ are fully allocated at or before $t^{k}$,

$$
r_{d_{S^{\prime \prime}}}\left(t^{k}\right)=\sum_{h \in H^{k-1} \cap\left(U_{S^{\prime \prime}} \backslash U_{S^{\prime}}\right)}\left(r_{h}\left(t^{k-1}\right)-\sum_{i \in M\left(h, H^{k-1}\right)_{t^{k-1}}} \int_{t^{k}}^{t^{k}} \omega_{i}(y) d y\right)-\sum_{i \in S^{\prime \prime}}\left(1-\int_{0}^{t^{k}} \omega_{i}(y) d y\right) .
$$


It implies that, for $S^{\prime} \cup S^{\prime \prime}$,

$$
\begin{aligned}
& \sum_{h \in H^{k-1} \cap U_{S^{\prime} \cup S^{\prime \prime}}}\left(r_{h}\left(t^{k-1}\right)-\sum_{i \in M\left(h, H^{k-1}\right)_{t^{k-1}}} \int_{h \in H^{k-1} \cap\left(U_{S^{\prime \prime}} \backslash U_{S^{\prime}}\right)}^{t^{k}} \omega_{i}(y) d y\right) \\
= & \left.\sum_{h}\left(t^{k-1}\right)-\sum_{i \in M\left(h, H^{k-1}\right)_{t^{k-1}}} \int_{i}^{t^{k}} \omega_{i}(y) d y\right) \\
& +\sum_{h \in H^{k-1} \cap U_{S^{\prime}}}\left(r_{h}\left(t^{k-1}\right)-\sum_{i \in M\left(h, H^{k-1}\right)_{t^{k-1}}} \int_{t^{k}} \omega_{i}(y) d y\right) \\
\leq & \sum_{i \in S^{\prime \prime}}\left(1-\int_{0}^{t^{k}} \omega_{i}(y) d y\right)+\sum_{i \in S^{\prime}}\left(1-\int_{0}^{t_{0}} \omega_{i}(y) d y\right)=\sum_{i \in S^{\prime} \cup S^{\prime \prime}}\left(1-\int_{0}^{t^{k}} \omega_{i}(y) d y\right)
\end{aligned}
$$

where the last equality follows from the fact that the sets $S^{\prime}$ and $S^{\prime \prime}$ are disjoint. This means that the slack of the IR constraint of $S^{\prime} \cup S^{\prime \prime}$ is non-positive at time $t^{k}$, thus

$$
r_{d_{S^{\prime} \cup S^{\prime \prime}}}\left(t^{k-1}\right)=\int_{t^{k-1}}^{t\left(S^{\prime} \cup S^{\prime \prime}\right)} \omega_{d_{S^{\prime} \cup S^{\prime \prime}}}(y) d y, \quad \text { where } t\left(S^{\prime} \cup S^{\prime \prime}\right) \leq t^{k}
$$

If this inequality is strict, then it contradicts the definition of $t^{k}$. If it holds with equality, then $S^{\prime} \cup S^{\prime \prime} \in B^{k}$. But it contradicts $S^{\prime \prime} \subseteq I_{E}^{k}$. QED

Now, we show that $Q(\omega)$ is individually rational. Let agent $i$ be an existing tenant. If he leaves the algorithm at time 1 , then, by Lemma 3 , he is assigned one unit from the houses in $U_{i}$; thus, the random assignment $Q(\omega)$ is individually rational for agent $i$. Let $i \in S$ for some $S \in B^{k}$ where $t^{k}<1$. By Lemma 3,

$$
\sum_{h \in H^{k-1} \cap U_{i}} r_{h}\left(t^{k-1}\right)>1-\int_{0}^{t^{k-1}} \omega_{i}(y) d y .
$$


Also, by definition of $t^{k}$,

$$
\sum_{h \in H^{k-1} \cap U_{i}}\left(r_{h}\left(t^{k-1}\right)-\sum_{i \in M\left(h, H^{k-1}\right)_{t^{k-1}}} \int_{t^{k}}^{t^{k}} \omega_{i}(y) d y\right) \geq 1-\int_{0}^{t^{k}} \omega_{i}(y) d y .
$$

Note that this holds with equality if, and only if, $\{i\} \in B^{k}$. In this case, the remainder of each house $h \in H^{k-1} \cap U_{i}$ is allocated to agent $i$, and agent $i$ is assigned a total of one unit. Otherwise, the houses in $H^{k-1} \cap U_{S}$ are allocated to the agents in $S$. From Lemma 1, we know that there is an allocation that is individually rational for each agent in $S$ (including $i$ ). The same argument applies recursively to the sub-problem $\left(S, t^{k}\right)$ : if agent $i$ has not been assigned one unit of houses, then the remainder of the houses in $U_{i}$ is enough to guarantee him a random consumption. The GE algorithm maintains this guarantee until the end and the induced random assignment $Q(\omega)$ is individually rational for agent $i$.

Now, we show that $Q(\omega)$ is ordinally efficient. We need the following characterization result:

Lemma 4 (Bogomolnaia and Moulin 2001) Given a preference profile $R$ and a random assignment $Q$, define a binary relation in $H$ as follows:

$$
\text { for each } h, h^{\prime} \in H: h \tau(Q, R) h^{\prime} \Leftrightarrow\left\{\text { there is } i \in I: h P_{i} h^{\prime} \text { and } q_{i h^{\prime}}>0\right\} \text {. }
$$

The random assignment $Q \in \mathcal{Q}$ is ordinally efficient if, and only if, the relation $\tau(Q, R)$ is acyclic.

Suppose the random assignment $Q(\omega)$ is not ordinally efficient. Then, by Lemma 4, there is a set of houses $\left\{h_{1}, h_{2}, \ldots, h_{k}\right\}$ and a function $\beta:\left\{h_{1}, h_{2}, \ldots, h_{k}\right\} \rightarrow I$ such that

$$
h_{1} P_{\beta\left(h_{1}\right)} h_{2} P_{\beta\left(h_{2}\right)} h_{3} P_{\beta\left(h_{3}\right)} \ldots h_{k} P_{\beta\left(h_{k}\right)} h_{1}
$$

and, for $i=1, \ldots, k-1, q(\omega)_{\beta\left(h_{i}\right), i+1}>0$, and $q(\omega)_{\beta\left(h_{k}\right), 1}>0$.

Let $t(h)$ denote the time at which house $h$ is allocated. At time $t\left(h_{1}\right)$, agent $\beta\left(h_{1}\right)$ eats either house $h_{1}$ or a better house for himself, and he has not yet eaten house $h_{2}$. Thus, $q(\omega)_{\beta\left(h_{1}\right), 2}>0$ implies $t\left(h_{1}\right) \leq t\left(h_{2}\right)$. By the same argument, $t\left(h_{1}\right) \leq t\left(h_{2}\right) \leq$ 
$\ldots \leq t\left(h_{k}\right) \leq t\left(h_{1}\right)$. Thus,

$$
t\left(h_{1}\right)=t\left(h_{2}\right)=\ldots=t\left(h_{k}\right)=t^{k} .
$$

At time $t \in\left[0, t^{k}\right)$, each house in $\left\{h_{1}, h_{2}, \ldots, h_{k}\right\}$ is available; for $i=1, \ldots, k-1$, $q(\omega)_{\beta\left(h_{i}\right), i+1}^{k-1}=0$ and $q(\omega)_{\beta\left(h_{k}\right), 1}^{k-1}=0$. Also, since

$$
\sum_{i \in M\left(h_{i}, H^{k-1}\right)_{t^{k-1}}} \int_{t^{k}}^{t^{k}} \omega_{i}(y) d y=r_{h_{i}}\left(t^{k-1}\right)
$$

implies $q(\omega)_{\beta\left(h_{i-1}\right), i}=0$, it must be that for each $i=1,2, \ldots, k$,

$$
\sum_{i \in M\left(h_{i}, H^{k-1}\right)} \int_{t^{k-1}}^{t^{k}} \omega_{i}(y) d y<r_{h_{i}}\left(t^{k-1}\right) .
$$

Thus, since houses $h_{1}, h_{2}, \ldots, h_{k}$ are allocated at time $t^{k}$, for each $i=1,2, \ldots, k$, $h_{i} \in U_{S}$ for some $S \in B^{k}$. Also, since house $h_{1}$ is allocated in the problem $\left(\bigcup_{S \in B^{k}} S, t^{k}\right)$, agent $\beta\left(h_{k}\right)$ has not eaten $h_{1}$ until $t^{k}$, and $q(\omega)_{\beta\left(h_{k}\right), 1}>0$, agent $\beta\left(h_{k}\right)$ must be in a bottleneck group $S \in B^{k}$. This holds for each agent $\beta\left(h_{i}\right)$ for $i=1, \ldots, k$; thus,

$$
\left\{\beta\left(h_{1}\right), \beta\left(h_{2}\right), \ldots, \beta\left(h_{k}\right)\right\} \subseteq\left(\bigcup_{S \in B^{k}} S\right) \subseteq I \backslash I_{E}^{k} .
$$

Let $S_{\beta\left(h_{i}\right)}^{k} \in B^{k}$ be the smallest bottleneck set that involves $\beta\left(h_{i}\right)$. We claim that $S_{\beta\left(h_{1}\right)}^{k} \subseteq S_{\beta\left(h_{2}\right)}^{k}$. First, suppose $S_{\beta\left(h_{1}\right)}^{k} \supseteq S_{\beta\left(h_{2}\right)}^{k}$. Then, $h_{2}$ will be allocated in the problem $\left(S_{\beta\left(h_{2}\right)}^{k}, t^{k}\right)$, and since $\beta\left(h_{1}\right) \notin S_{\beta\left(h_{2}\right)}^{k}, q(\omega)_{\beta\left(h_{1}\right), 2}=0$, which is a contradiction. Now, suppose that $S_{\beta\left(h_{1}\right)}^{k} \nsubseteq S_{\beta\left(h_{2}\right)}^{k}$ and $S_{\beta\left(h_{1}\right)}^{k} \nsupseteq S_{\beta\left(h_{2}\right)}^{k}$. Since, by Lemma $2, S_{\beta\left(h_{1}\right)}^{k} \cap S_{\beta\left(h_{2}\right)}^{k}$ (if nonempty) is a bottleneck set, it must be that $\beta\left(h_{1}\right) \in S_{\beta\left(h_{1}\right)}^{k} \backslash S_{\beta\left(h_{2}\right)}^{k}$ and $\beta\left(h_{2}\right) \in$ $S_{\beta\left(h_{2}\right)}^{k} \backslash S_{\beta\left(h_{1}\right)}^{k}$. Thus,

$$
h_{2} \in U_{\left.S_{\beta\left(h_{1}\right)}^{k}\right) S_{\beta\left(h_{2}\right)}^{k}} \cap U_{S_{\beta\left(h_{2}\right)}^{k} \backslash S_{\beta\left(h_{1}\right)}^{k}} .
$$

If $h_{2} \in U_{S_{\beta\left(h_{1}\right)}^{k} \cap S_{\beta\left(h_{2}\right)}^{k}}$, then, by Lemma $2, S_{\beta\left(h_{1}\right)}^{k} \cap S_{\beta\left(h_{2}\right)}^{k}$ is a bottleneck set and $h_{2}$ is allocated to the agents in $S_{\beta\left(h_{1}\right)}^{k} \cap S_{\beta\left(h_{2}\right)}^{k}$. Then, since $\beta\left(h_{1}\right) \notin S_{\beta\left(h_{2}\right)}^{k}, q(\omega)_{\beta\left(h_{1}\right), 2}=0$, 
which is a contradiction. Thus, $h_{2} \notin U_{S_{\beta\left(h_{1}\right)}^{k} \cap S_{\beta\left(h_{2}\right)}^{k}}$, and

$$
h_{2} \in\left(U_{S_{\beta\left(h_{1}\right)}^{k} \backslash S_{\beta\left(h_{2}\right)}^{k}} \cap U_{S_{\beta\left(h_{2}\right)}^{k} \backslash S_{\beta\left(h_{1}\right)}^{k}}\right) \backslash U_{S_{\beta\left(h_{1}\right)}^{k} \cap S_{\beta\left(h_{2}\right)}^{k}} .
$$

Then, by equality (1) in the proof of Lemma 2,

$$
\sum_{i \in M\left(h_{2}, H^{k-1}\right.} \int_{t^{k-1}}^{t^{k}} \omega_{i}(y) d y=r_{h_{2}}\left(t^{k-1}\right)
$$

which is a contradiction. Thus, $S_{\beta\left(h_{1}\right)}^{k} \subseteq S_{\beta\left(h_{2}\right)}^{k}$. Since the same argument applies to $S_{\beta\left(h_{3}\right)}^{k}, S_{\beta\left(h_{4}\right)}^{k}, \ldots$, it implies

$$
S_{\beta\left(h_{1}\right)}^{k} \subseteq S_{\beta\left(h_{2}\right)}^{k} \subseteq \cdots \subseteq S_{\beta\left(h_{k}\right)}^{k} \subseteq S_{\beta\left(h_{1}\right)}^{k}
$$

Thus, $S_{\beta\left(h_{1}\right)}^{k}=S_{\beta\left(h_{2}\right)}^{k}=\cdots=S_{\beta\left(h_{k}\right)}^{k}=S_{1}$. The houses $h_{1}, h_{2}, \ldots, h_{k}$ are allocated to the agents in the sub-problem $\left(S_{1}, t^{k}\right)$. In the sub-problem $\left(S_{1}, t^{k}\right)$, the same argument applies: The houses $h_{1}, h_{2}, \ldots h_{k}$ and the agents $\beta\left(h_{1}\right), \beta\left(h_{2}\right), \ldots, \beta\left(h_{k}\right)$ leave at the same time, say time $t^{l}$. Let $S_{\beta\left(h_{i}\right)}^{l} \in B^{l}$ be the smallest bottleneck set that involves $\beta\left(h_{i}\right)$. Then, $S_{\beta\left(h_{1}\right)}^{l}=S_{\beta\left(h_{2}\right)}^{l}=\cdots=S_{\beta\left(h_{k}\right)}^{l}=S_{2}$. The houses $h_{1}, h_{2}, \ldots, h_{k}$ will be allocated to the agents in $S_{2}$ in the sub-sub-problem $\left(S_{2}, t^{l}\right)$. This contradicts the finiteness of problem.

Proof of Part 2: Let $Q$ be an individually rational and ordinally efficient random assignment. By Theorem 1, there exists a profile of eating speeds $\omega$ such that $Q$ is equivalent to the random assignment generated by the eating algorithm for $\omega$. Since $Q$ is individually rational, by Lemma 1 , the slack of each IR constraint is nonnegative throughout the algorithm. Now, consider the generalized eating algorithm. Since the slack of each IR constraint is nonnegative on $[0,1]$, there is no bottleneck set. Thus, the generalized eating algorithm reduces to the eating algorithm, and $Q(\omega)=Q$.

\section{PROOF OF PROPOSITION 1}

Let $\left(\mu^{0}, R\right)$ be a problem. Let $i \in I$ and label houses in $H$, such that

$$
h_{1} P_{i} h_{2} P_{i} \ldots P_{i} h_{\left|U_{i}\right|-1} P_{i} \mu^{0}(i)
$$


For each $i^{\prime} \in I$, let $k\left(i^{\prime}\right)$ be such that $i^{\prime} \in I^{k\left(i^{\prime}\right)-1} \backslash I^{k\left(i^{\prime}\right)}$. Let $i, j \in I$.

Case 1: $k(i)>k(j)$

The houses in $U_{j}$ are allocated at time $t^{k(j)} \leq t^{k(i)-1}$. Let $h$ be agent $i^{\prime}$ s favorite house among the houses in $H^{k(i)-1}$. Since $\mathrm{PS}_{i, h}^{\mathbf{I R}}>0$ and $h \notin U_{j}$, the random consumption $\mathrm{PS}_{i}^{\mathbf{I R}}$ is not individually rational for agent $j$.

Case 2: $k(i)<k(j)$

Let $S \in B^{k(i)}$ where $i \in S$. Each house in the set $U_{i}=\left\{h_{1}, h_{2}, \ldots, h_{\left|U_{i}\right|-1}, h_{i}\right\}$ is allocated at or before $t^{k(i)}$. Let $k_{1}$ be the step at which $h_{1}$ is allocated. Thus, $h_{1} \in H^{k_{1}-1} \backslash H^{k_{1}}$. Since $i \in M\left(h_{1}, H^{k}\right)$ for $k \leq k_{1}-1, q_{i 1}^{k_{1}} \geq t^{k_{1}}$. Also, $k\left(i^{\prime}\right)>k_{1}$ implies $t^{k_{1}} \geq q_{i^{\prime} 1}^{k_{1}}$. Since $k_{1} \leq k(i)<k(j), q_{i 1}^{k_{1}} \geq t^{k_{1}} \geq q_{j 1}^{k_{1}}$. Let $k_{2}$ be the step at which the houses $h_{1}$ and $h_{2}$ are allocated. Note that $k \leq k_{2}-1$ implies $i \in M\left(h_{1}, H^{k}\right) \cup M\left(h_{2}, H^{k}\right)$. Thus, $q_{i 1}+q_{i 2}=q_{i 1}^{k_{2}}+q_{i 2}^{k_{2}} \geq t^{k_{2}}$. Also, $k\left(i^{\prime}\right)>k_{2}$ implies $t^{k_{2}} \geq q_{i^{\prime} 1}^{k_{2}}+q_{i^{\prime} 2}^{k_{2}}$. Since $k_{2} \leq k(i)<k(j)$,

$$
q_{i 1}+q_{i 2}=q_{i 1}^{k_{2}}+q_{i 2}^{k_{2}}=t^{k_{2}} \geq q_{j 1}^{k_{2}}+q_{j 2}^{k_{2}}=q_{j 1}+q_{j 2} .
$$

The same argument applies to the rest of the houses in $U_{i}$. Thus, $\operatorname{PS}_{i}^{\mathbf{I R}}$ stochastically dominates $\mathrm{PS}_{j}^{\mathbf{I R}}$ at $R_{i}$, and agent $i$ does not envy agent $j$.

Case 3: $k(i)=k(j)=k^{*}$

If $t^{k^{*}}=1$, then, by the same argument used in the proof of Proposition 1 by Bogomolnaia and Moulin (2001), agent $i$ does not envy agent $j$. Assume $t^{k^{*}}<1$. Thus, $i, j \in$ $\bigcup_{S \in B^{k^{*}}} S$. We now consider the problem $\left(\bigcup_{S \in B^{k^{*}}} S, t^{k^{*}}\right)$. Let $S_{i}, S_{j} \subseteq \bigcup_{S \in B^{k^{*}}} S$ be the smallest bottleneck sets involving $i$ and $j$, respectively.

If $S_{i} \cap S_{j}=\emptyset$, then by Lemma 2 ,

$$
\left(H^{k^{*}-1} \backslash\left\{h: t^{k^{*}}=t^{k^{*}}(h)\right\}\right) \cap\left(U_{S_{i}} \cap U_{S_{j}}\right)=\emptyset .
$$

Thus, the random consumption $\mathrm{PS}_{i}^{\mathbf{I R}}$ is not individually rational for agent $j$.

If $S_{i} \neq S_{j}$ and $S_{i} \cap S_{j} \neq \emptyset$, then either $i \in S_{i} \backslash S_{j}$ or $j \in S_{j} \backslash S_{i}$. At time $t^{k^{*}}$, the remainder of the houses in $U_{S_{i} \cap S_{j}}$ will be allocated to the bottleneck group $S_{i} \cap S_{j}$. Let $H^{\prime}=\left(U_{S_{i} \backslash S_{j}} \cap U_{S_{j} \backslash S_{i}}\right) \backslash U_{S_{i} \cap S_{j}}$. Since, by equality (1) in the proof of Lemma 2,

$$
\sum_{h \in H^{k^{*}-1} \cap H^{\prime}}\left(r_{h}\left(t^{k^{*}-1}\right)-\left(t^{k^{*}}-t^{k^{*}-1}\right) m\left(h, H^{k^{*}-1}\right)\right)=0 .
$$


the remaining houses in $U_{S_{i} \backslash S_{j}}$ and $U_{S_{j} \backslash S_{i}}$ are disjoint. Thus, if $i \in S_{i} \backslash S_{j}$, then $\mathrm{PS}_{i}^{\mathbf{I R}}$ is not individually rational for agent $j$. If $i \notin S_{i} \backslash S_{j}$ and $j \in S_{j} \backslash S_{i}$, then, since $S_{j} \backslash S_{i}$ is not a bottleneck set, agent $i$ leaves before agent $j$. Then, by the same argument in Case $2, \mathrm{PS}_{i}^{\mathbf{I R}}$ stochastically dominates $\mathrm{PS}_{j}^{\mathbf{I R}}$.

If $S_{i}=S_{j}=S$, then, without loss of generality, we assume that, in the problem $\left(S, t^{k^{*}}\right)$, agents $i$ and $j$ leave the algorithm at time $1-t^{k^{*}}$. (If not, then, in the problem $\left(S, t^{k^{*}}\right)$, either Case 1 or Case 2 applies, thus, either $\mathrm{PS}_{i}^{\mathbf{I R}}$ is not individually rational for agent $j$, or $\mathrm{PS}_{i}^{\mathbf{I R}}$ stochastically dominates $\mathrm{PS}_{j}^{\mathbf{I R}}$ at $R_{i}$.) Then agent $i$ does not envy agent $j$ by the same argument in the proof of Proposition 1 by Bogomolnaia and Moulin (2001).

\section{PROOF OF THEOREM 4}

First note that it is enough to consider the case of three agents: For an arbitrary number of agents $|I|$, let agents $1,2,3$ prefer $h_{1}, h_{2}$, and $h_{3}$ to other houses, while for $i>3$, agent $i$ is the existing tenant of $h_{i} \neq h_{0}$, and $h_{i} P_{i} h_{j}$ for $j=1,2,3$. By individual rationality, the houses $h_{4}, h_{5}, \ldots, h_{|I|}$ are allocated to the agents $4,5, \ldots,|I|$, and the houses $h_{1}, h_{2}, h_{3}$ are allocated to the agents $1,2,3$.

Let the endowment profile be such that $\mu^{0}(1)=h_{1}, \mu^{0}(2)=h_{2}$, and $\mu^{0}(3)=h_{0}$. Let $\varphi$ be a solution satisfying individual rationality, strategy-proofness and no justified-envy. We proceed by considering three preference profiles. Let $\varphi_{i j}^{k}$ represent the probability that agent $i$ is assigned house $h_{j}$ under the preference profile $k$.

$$
\begin{array}{ccccccccc}
\frac{R_{1}}{h_{2}} & \frac{R_{2}}{h_{1}} & \frac{R_{3}}{h_{2}} & \frac{R_{1}}{h_{2}} & \frac{R_{2}}{h_{1}} & \frac{R_{3}}{h_{2}} & \frac{R_{1}}{h_{2}} & \frac{R_{2}}{h_{1}} & \frac{R_{3}}{h_{2}} \\
h_{1} & h_{2} & h_{1} & h_{3} & h_{2} & h_{1} & h_{3} & h_{3} & h_{1} \\
h_{3} & h_{3} & h_{3} & h_{1} & h_{3} & h_{3} & h_{1} & h_{2} & h_{3}
\end{array}
$$

Profile 1: By individual rationality, $h_{1}$ and $h_{2}$ are allocated to agents 1 and 2. Note that $\varphi_{12}^{1}=\varphi_{21}^{1}$. By no justified-envy, $\varphi_{12}^{1}=\varphi_{21}^{1} \geq 1 / 2$.

Profile 2: By strategy-proofness, $\varphi_{12}^{2} \geq \varphi_{12}^{1} \geq 1 / 2$. By no justified-envy, $\varphi_{12}^{2}=\varphi_{32}^{2}$. Thus, $\varphi_{12}^{2}=\varphi_{32}^{2}=1 / 2$. By individual rationality, $\varphi_{21}^{2}=1$.

Profile 3: By strategy-proofness, $\varphi_{21}^{3}=1$. By no justified-envy, agent 3 does not envy agent 2 , implying that $\varphi_{32}^{3}+\varphi_{31}^{3} \geq \varphi_{22}^{3}+\varphi_{21}^{3}=1$. Thus, $\varphi_{32}^{3}=1$ and $\varphi_{13}^{3}=1$. However, at this allocation, agent 1 envies agent 3 , and this contradicts no justified-envy. This 
proves the first part of the theorem.

For the second part, note that, by individual rationality and ordinal efficiency, $\varphi_{12}^{1}=\varphi_{21}^{1}=1$. By weak strategy-proofness, $\varphi_{12}^{2}=1$. Thus, $\varphi_{1}^{2} s d\left(R_{i}\right) \varphi_{3}^{2}$ and $\varphi_{1}^{2} \neq \varphi_{3}^{2}$ and $\operatorname{Support}\left(\varphi_{3}^{2}\right) \backslash U_{1}=\emptyset$. But this violates weak no justified-envy.

\section{PROOF OF PROPOSITION 2}

Let $\left(\mu^{0}, R\right)$ be a problem, where $\left\{j \in I_{E}: U_{j}=H\right\} \neq \emptyset$. Let

$$
k^{*}=\operatorname{ArgMax}\left\{k: t^{k}<1 \text { and } B^{k} \neq \emptyset\right\} .
$$

If $k^{*}$ is not well defined, then each agent leaves at time 1. Then, individual rationality does not restrict the set of random assignments and for the problem $\left(\mu^{1}\left(\mu^{0}, R\right), R\right)$, the $\mathrm{PS}^{\mathbf{I R}}$ solution gives the same random assignment. Suppose $k^{*}$ is well defined. We claim that for each $k \in\left\{0,1 \ldots, k^{*}\right\}$ and for each $S \in B^{k},\left\{j \in I_{E}: U_{j}=H\right\} \cap S=\emptyset$. Suppose not. Then, there is $k_{1}$ such that $\left\{j \in I_{E}: U_{j}=H\right\} \cap S \neq \emptyset$ for some $S \in B^{k_{1}}$. Since $S$ involves an agent $i$ such that $U_{i}=H$, and $\left(H^{k_{1}-1} \backslash\left\{h: t^{k_{1}}=t^{k_{1}}(h)\right\}\right) \subset U_{S}$, all the remainders of the houses are allocated and the algorithm terminates at time $t^{k_{1}}$. Since $I_{N} \subset I^{k^{*}}$, it implies $I_{N}=\emptyset$ and $k_{1}=k^{*}$. Then, since the algorithm terminates at $t^{k^{*}}<1$,

$$
t^{k^{*}}=t^{k^{*}-1}+\frac{r_{I_{E}^{k^{*}-1}}\left(t^{k^{*}-1}\right)}{\omega_{I_{E}^{k^{*}-1}}\left(t^{k^{*}-1}\right)} .
$$

But, this contradicts $\omega_{I_{E}^{k^{*}-1}}\left(t^{k^{*}-1}\right)=0$. Thus, for each $k \in\left\{0,1 \ldots, k^{*}\right\}$ and for each $S \in B^{k},\left\{j \in I_{E}: U_{j}=H\right\} \cap S=\emptyset$. Thus, $\left\{j \in I_{E}: U_{j}=H\right\} \subset I_{E}^{k^{*}}$ and the algorithm terminates at $t=1$. Each agent in $\left\{j \in I_{E}: U_{j}=H\right\}$ has eaten 1 unit through $[0,1)$. Thus, the PS ${ }^{\mathbf{I R}}$ solution is invariant to whether an agent is an existing tenant of the bottom house in his ranking or he is a new applicant.

\section{REFERENCES}

Abdulkadiroğlu, Atila, and Tayfun Sönmez, 1998. Random Serial Dictatorship and the Core from Random Endowments in House Allocation Problems. Econometrica 66, 689-701.

Abdulkadiroğlu, Atila, and Tayfun Sönmez, 1999. House Allocation with Existing Tenants. Journal of Economic Theory 88, 233-260 
Abdulkadiroğlu, Atila, and Tayfun Sönmez, 2003. Ordinal Efficiency and Dominated Sets of Assignments. Journal of Economic Theory 112, 157-172.

Abdulkadiroğlu, Atila, and Tayfun Sönmez, 2003. School Choice: A Mechanism Design Approach. American Economic Review 93, 729-747.

Birkhoff, Garrett, 1946. Tres Observaciones Sobre el Algebra Lineal. Univ. Nac. Tucuman Rev. Ser. A 5, 147-151.

Bogomolnaia, Anna, and Hervé Moulin, 2001. A New Solution to the Random Assignment Problem. Journal of Economic Theory 100, 295-328.

Bogomolnaia, Anna, and Hervé Moulin, 2002. A Simple Random Assignment Problem with a Unique Solution. Economic Theory 19, 623-636.

Crès, Hérve, and Hervé Moulin, 2001. Scheduling with Opting Out: Improving upon Random Priority. Operations Research 49, 565-576.

Ford, Lester R., and Delbert R. Fulkerson, 1971. Flows in Networks. Princeton, New Jersey: Princeton University Press.

Gale, David, 1957. A Theorem on Flows in Networks. Pacific Journal of Mathematics 7, 1073-1082.

Hall, Philip, 1935. On Representatives of Subsets. Journal of Mathematical Society 19, 26-30.

Hylland, Aanund, and Richard J. Zeckhauser, 1979. The Efficient Allocation of Individuals to Positions. Journal of Political Economy 87, 293-314.

Kojima, Fuhito, and Mihai Manea, 2007. Strategy-proofness of the Probabilistic Serial Mechanism in Large Random Assignment Problems. http://www.people.fas.harvard.edu/ kojima/papers.html.

Lovász, László, and Michael D. Plummer, 1986. Matching Theory. Amsterdam, New York: North-Holland Press.

Ma, Jingpeng, 1994. Strategy-proofness and Strict Core in a Market with Indivisibilities. International Journal of Game Theory 23, 75-83.

McLennan, Andrew, 2002. Ordinal Efficiency and the Polyhedral Separating Hyperplane Theorem. Journal of Economic Theory 105, 435-449.

Pápai, Szilvia, 2000. Strategyproof Assignment by Hierarchical Exchange. Econometrica 68, 1403-1434.

Pathak, Parag A., 2006. Lotteries in Student Assignments. http://www.people.fas.harvard.edu/ ppathak. 
Roth, Alvin E., 1982. Incentive Compatibility in a Market with Indivisible Goods. Economic Letters 9, 127-132.

Roth, Alvin E., and Andrew Postlewaite, 1977. Weak versus Strong Domination in a Market with Indivisible Goods. Journal of Mathematical Economics 4, 131-137.

Roth, Alvin E., and Uriel G. Rothblum, 1999. Truncation Strategies in Matching Markets: In Search of Advice for Participants. Econometrica 67, 21-44.

Roth, Alvin E., and Marilda Sotomayor, 1990. Two Sided Matching: A Study in Game-Theoretic Modeling and Analysis. Cambridge: Cambridge University Press.

Roth, Alvin E., Tayfun Sönmez, and M. Utku Ünver, 2004. Kidney Exchange. Quarterly Journal of Economics 119, 457-488.

Sethuraman, Jay, 2001. A New Solution to the House Allocation Problem with Existing Tenants. Unpublished mimeo. University of Columbia.

Shapley, Lloyd S., and Herbert Scarf, 1974. On Cores and Indivisibility. Journal of Mathematical Economics 1, 23-27.

Sönmez, Tayfun, and M. Utku Ünver, 2005. House Allocation with Existing Tenants: An Equivalence. Games and Economic Behavior 52, 153-185.

Svensson, Lars-Gunnar, 1999. Strategy-Proof Allocation of Indivisible Goods. Social Choice and Welfare 16, 557-567.

Thomson, William, Forthcoming. The Theory of Fair Allocation. Princeton, New Jersey: Princeton University Press.

Von Neumann, John, 1953. A Certain Zero-sum Two-person Game Equivalent to the Optimal Assignment Problem. In: Harold W. Kuhn and Albert W. Tucker (Eds.), Contribution to the Theory of Games. Princeton, New Jersey: Princeton University Press, pp. 5-12.

Yılmaz, Özgür, Forthcoming. Random Assignment under Weak Preferences. Games and Economic Behavior.

Zhou, Lin, 1990. On a Conjecture by Gale About One-Sided Matching Problems. Journal of Economic Theory 52, 123-135. 